\title{
Concepts of the new ASDEX Upgrade flight simulator
}

\author{
W. Treutterer, E. Fable, A. Gräter, F. Janky, O. Kudlacek, I. Gomez Ortiz, T. Maceina, G. Raupp, \\ B. Sieglin, T. Zehetbauer, ASDEX Upgrade Team ${ }^{\mathrm{a}}$
}

${ }^{a}$ Max-Planck-Institute for Plasma Physics, Boltzmannstraße 2, D-85748 Garching, Germany

\begin{abstract}
Discharge scenarios and control schemes in ASDEX Upgrade (AUG) are evolving more and more complex. Especially in physics investigations for ITER and DEMO sophisticated scenarios exploit the operational space. This increases the probability of design flaws or human errors in the pulse configuration, but also aggravates the potential damage in the failure case.

The ASDEX Upgrade Flight Simulator Fenix, which is currently under construction, will provide a fast and efficient simulation tool for testing and validating discharge scenarios, as well as control and monitoring functions, during their development and immediately prior to experimental pulse execution. This ensures, that the scenarios and settings are adequate to reach the experimental goals and that the margins to operational limits are sufficiently large also during the dynamic evolution of the discharge. Simplified physics and plant system "control" models combined with a representation of the ASDEX Upgrade Discharge Control System (DCS) allow for fast simulation runs with reasonable prediction quality. In the simulation an event generator can trigger plasma instabilities, technical failures and external events to test the resilience of the designed pulse against unplanned incidents. The granularity of modelling shall be customizable, such that the simulator can also be used for detail investigations with elaborate physics at the cost of longer simulation time.

As a basis for implementation the ITER Plasma Control System Simulation Platform (PCSSP) has been chosen. The flight simulator, extends PCSSP with an ASTRA co-simulator for the ASDEX Upgrade tokamak model and with custom modules for its actuators, diagnostics and control system. Plugins will enable reading original AUG discharge programs and configuration files, as well as storing the results in the AUG shot file database.
\end{abstract}

Keywords: plasma control, investment protection, simulation, control oriented modeling, ASDEX Upgrade

\section{Introduction}

ASDEX Upgrade is a mid-size tokamak device with a large number of actuators (11 Power supplies, 18 heating systems, 20 gas channels and a pellet centrifuge). Current research goals comprise among others MHD control, heat flux and detachment control, pellet fueling, disruption avoidance and mitigation, scenario development and control system design - often also with respect to the investigation of ITER and DEMO aspects[1,2]. Moreover, many of these topics cannot be addressed isolated as the physics effects are strongly coupled. Control of such complex experiments requires a versatile control system with lots of configuration knobs and a tailored evolution of plasma state (scenario).

Given the complexity, the success of an experiment depends increasingly on the consistence of configuration, control system functions, scenario and underlying assumptions on the plasma behavior. Poorly designed pulse schedule references, editing oversights, or immature control algorithms are likely to baffle reaching the pulse goals or even cause premature discharge termination. Prior simulation of the plasma control system and the tokamak lends itself as an attractive solution to check the consistency in advance, thereby avoiding time, staff and money expensive experimental iterations. Simulation can also help to analyze the risk potential of operating close to technical or plasma physical limits and to develop control and protection strategies allowing to safely exploit such regions and reduce fatigue of machine components.
To be effective, the simulation must not only be run frequently during pulse schedule development or control design, but most importantly shortly before pulse execution. Thus, simulation of very detailed models taking hours is not applicable. Instead, short evaluation times are desired, where ideally simulation would be (faster-than) real-time. This is currently only achievable by the use of simplified, control-oriented physics models. Such simulators are often attributed as "flight simulators".

Starting with magnetic equilibrium control [3-6], model based control design has evolved also into kinetic and MHD control [7-10]. This kind of models, tailored to the respective fusion devices form the backbone of flight simulators. Flight simulators, however, require also a well-developed simulation engine, user interface, automation API and integration with domain-specific configuration data sources. Already in 2005, development of AUGFS, a flight simulator based on Modelica [11] and Dymola [12] had been initiated at ASDEX Upgrade [13], but was discontinued later on. In the same year, DINA$\mathrm{CH}$ [14] emerged with a DINA model of the TCV tokamak implemented on the Simulink ${ }^{\circledR}$ platform [15]. ToreSupra based its GMFS flight simulator on a SciCosKepler framework [16,17]. The TokSys environment developed at DIII-D [18] and implemented in Simulink ${ }^{\circledR}$ the gave inspiration to ITER's Plasma Control System Simulation Platform (PCSSP) [19-22], which is continuously being developed further and customizable to any fusion device. 
PCSSP is also the base of a new approach for an ASDEX Upgrade flight simulator: Fenix. Its construction is in progress and follows the concepts that are outlined in the subsequent sections. Details of the implementation, as well as first simulation results, are published in a separate contribution [23], however. Section 2 explains the envisaged use of the flight simulator in ASDEX Upgrade's experiment preparation process, while section 3 describes the main components. Finally, section 4 informs about the current state of the project, next steps and future plans.

\section{Workflow integration}

In preparation of experiments at ASDEX Upgrade an experiment leader specifies the Discharge Program (DP) references, defining the pulse scenario. In parallel, control system properties are checked and, if necessary, settings are adjusted and features are amended or developed newly. Without the flight simulator, only simple validation checks can be performed, verifying for instance, that the programmed plasma and PF coil reference currents stay within their operational limits. As the behavior of plasma and plant systems is not considered, more subtle design flaws such as an undesired reference waveform, still within the operational limits, which can be a left-over from a template pulse, or an unintentional approach to a stability limit remain undetected. When the experiment is executed, it will most probably fail to reach its target and needs to be repeated after adjusting DP or control system settings - often in a series of iterations. With the flight simulator, heavyweight experiment iterations involving many systems and staff will be transformed into light-weight design iterations performed by a developer as shown in figure 1 .

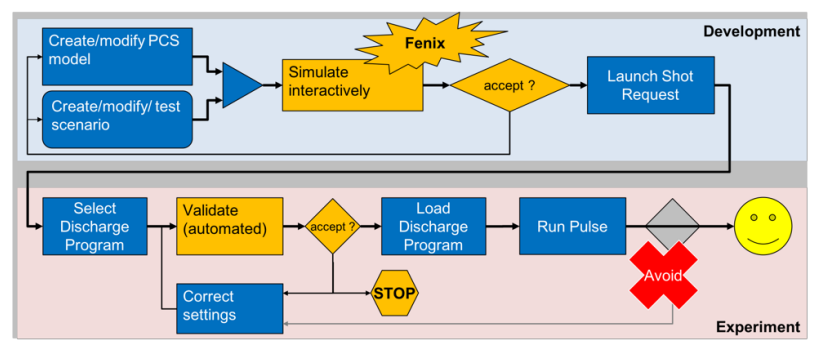

Figure 1: Experiment preparation and PCS development workflow with Fenix

During DP or control system development, the developer will repeatedly run simulations, also deliberately adding disturbing events, in order to validate his design and estimating its robustness. In interactive mode, the flight simulator offers a variety of support functions to analyze and assess the behavior of the entire system: time trace display of quantities of interest, which would not even be measurable in the real experiment, sensitivity analysis at selected operation points, parameter scans, etc. The developer will request shot execution only after the simulation results satisfy the acceptance criteria, thus eliminating the most frequent errors in DP and control system settings. In its first version, Fenix will implement acceptance criteria for operational boundaries as well as margins to actuator saturation and stability limits. Future versions will allow users to specify success conditions, confirming that the planned pulse can reach its experimental goal.

When, however, the experiment is going to be executed, the actual plant system settings might differ from what the developer had assumed during the design. ASDEX Upgrade's agile experimentation policies also permit DP variations during shot execution preparations. As a protection provision, the flight simulator shall therefore also be run automatically with the active plant and control system settings as a part of the pulse countdown, such as to validate the final settings. For this purpose, Fenix must be able not only to read the DP but also the genuine settings of plasma control system and plant systems. The automated check requires formalized acceptance criteria yielding an unambiguous result status that allows to run the pulse or to deny its execution. In the latter case, the experiment leader or responsible technical officers still can override the negative recommendation and run the pulse nevertheless, or they can correct the settings, and another validation can be attempted.

\section{Components}

PCSSP [21], the ITER simulation framework, has been chosen as the foundation for Fenix implementation. It is customized with a model of the ASDEX Upgrade DCS control system [24] and access to all relevant configuration sources. A connected ASTRA-SPIDER $[25,26]$ co-simulation process computes the ASDEX Upgrade tokamak, and the physics aspects of its actuators and diagnostics, re-using a development originally made for DEMO studies [27] (figure 2).

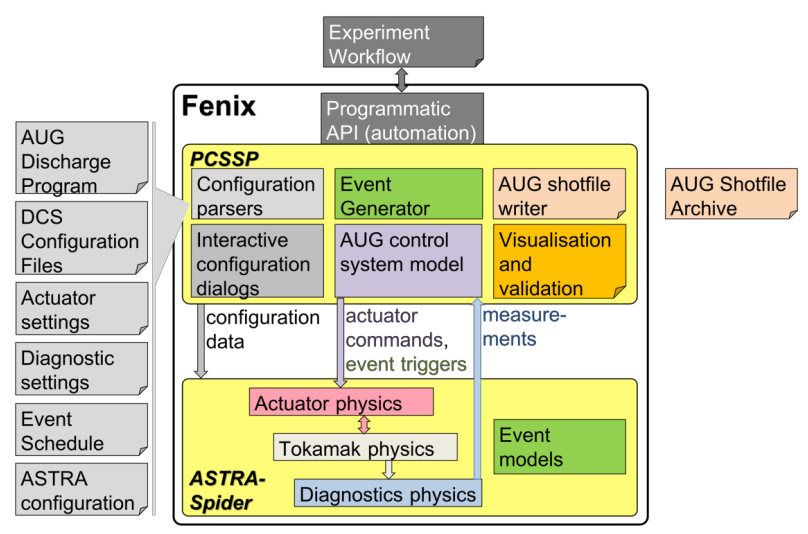

Figure 2: Fenix components with main function modules

\subsection{PCSSP}

PCSSP is built on MATLAB/Simulink and thus can make use of their intrinsic functionalities, especially the user interface, a graphical model editor, model block libraries, toolboxes for control system design, visualization tools, etc. On top of that PCSSP provides block libraries dedicated for plasma control system modeling, tokamak modeling and event generation, along with administrative framework functions for configuration data, results archiving, block connection and documentation. All elements are highly customizable 
allowing easy adaptation to any context. Moreover, the open architecture enables users to add their own modules.

Fenix can directly use PCSSP's Event and Reference Generator blocks with built-in exception handling, as well as generic feedback controller blocks (compact controller). Furthermore, custom blocks need to be developed for Fenix, modeling the DCS control system, as well as control system interfaces of actuator and diagnostic systems. As the configuration of these blocks shall use the same sources as the real systems, an interface to the ASDEX Upgrade parameter server will be provided, that does not only return actual settings but can also deliver settings from previous archived shots or predefined standard parameters. At a lower level it is also possible to directly parse DCS configuration files, e.g. those containing settings of the feedback controllers. Finally, a PCSSP archiving plugin for storing simulation results to the AUG shotfile archive format will be supplied.

While typically an entire system is modelled in PCSSP, also external simulation codes can be coupled with Simulink's S-function interface and run in cosimulation mode. Thus, existing, proprietary or very detailed models can be attached exploiting the strengths of both, the modeling detail of the external code and the framework benefits of PCSSP.

Fenix takes advantage from this feature, using the external ASTRA-SPIDER code to co-simulate all physics aspects of ASDEX Upgrade including tokamak, actuator and diagnostic components. For this purpose an Sfunction developed earlier for DEMO modelling [27] has been adapted.

\subsection{ASTRA-SPIDER}

ASTRA [25] is a code for modelling 1-D particle transport, i.e. kinetic temperature and density profiles as functions of the magnetic flux coordinate $\rho$ tor. This simplifying assumption is adequate for the purpose of the flight simulator and leverages fast simulation at still reasonable accuracy. On demand, the ASTRA model can be extended with greater detail such as an MHD module, which, however, increases the simulation time.

The dynamic variability of the magnetic equilibrium, i.e. the plasma current, current profile and shape is contributed by SPIDER [28]. In addition to solving the Grad-Shafranov equation, SPIDER comprises a model of the magnetic coils. Both codes work in tight crosscoupling to model the interaction of particle temperature and pressure with magnetic flux profile appropriately.

At this stage actuator and diagnostics are modelled as ideal systems. Heating or current drive power requests, from the control system are delivered without losses to the plasma. The fueling model comprises a gas flow propagating with some delay through the gas pipes, as well as pellet trains at discrete centrifuge frequencies. Ideal diagnostics are delivering the actual plasma quantities for control.
The flexibility of the PCSSP platform, however, allows to add refined models or disturbance sources, such as noise at any later time.

\subsection{Results evaluation}

In interactive mode PCSSP's and Simulink's built-in scopes and gauges, as well as ASTRA's flux surface visualization allow to observe the evolution of control and modeled physics variables already while the simulation is running. As the results are stored in ASDEX Upgrade shotfile format, they can further be analyzed with any existing Matlab graphics tools and ASDEX Upgrade postshot analysis applications.

The flight simulator, however, shall also return a statement on the eligibility of an experiment setup for execution given the modeled device and control system. For example, it can detect undesired steps in reference waveforms stemming from left-over data points of an earlier boilerplate. This can be accomplished by automated post-simulation analysis of the dedicated time traces. User-defined acceptance criteria shall ensure that the values of all key variables remain within given bounds, that can be static or dependent on other simulated plasma and plant state variables. For this purpose, Fenix will feature a generic validation module, where users can configure their acceptance criteria. It will return a success indicator that can be evaluated by the experiment workflow control in order to continue or block the further preparation of the envisaged pulse.

\section{Outlook}

Currently, Fenix is still under construction. The first version of ASDEX Upgrade physics model in ASTRASimulink is complete. Likewise, core control system components such as the reference generator and the feedback controller model and a model of the AUG coil power supplies are already in place. Thus, first simulations have already been conducted demonstrating the essential capabilities of the flight simulator [23].

Next, control system component models for discharge monitoring, the configuration from the experiment data sources, the AUG shotfile writer and the evaluation module will be implemented and the system will be tuned for speed and performance. Completion and integration in the ASDEX Upgrade experimentation workflow is envisaged until mid 2019.

In future, Fenix will not only optimize AUG experiment efficiency but with quick turnaround times allow rapid prototyping of new control system functions and boost the design of features like plasma profile control or actuator management.

\section{Acknowledgements}

This work has been carried out within the framework of the EUROfusion Consortium and has received funding from the Euratom research and training programme 20142018 under grant agreement No 633053. The views and 
opinions expressed herein do not necessarily reflect those of the European Commission.

\section{References}

[1] A. Kallenbach et al., Recent ASDEX Upgrade Results and Future Extension Plans, IEEE Trans. Plasma Sci. 40 (2012) 605-613. doi:10.1109/TPS.2011.2179068.

[2] Kallenbach.A. et al., Overview of ASDEX upgrade results, Nucl. Fusion. 57 (2017) 102015. doi:10.1088/1741-4326/aa64f6.

[3] R.R. Khayrutdinov, V.E. Lukash, Studies of Plasma Equilibrium and Transport in a Tokamak Fusion Device with the Inverse-Variable Technique, J. Comput. Phys. 109 (1993) 193-201. doi:10.1006/JCPH.1993.1211.

[4] R. Albanese, G. Calabrò, M. Mattei, F. Villone, Plasma response models for current, shape and position control in JET, Fusion Eng. Des. 66-68 (2003) 715-718. doi:10.1016/S0920-3796(03)00285-0.

[5] G. De Tommasi et al., XSC Tools: A Software Suite for Tokamak Plasma Shape Control Design and Validation, IEEE Trans. Plasma Sci. 35 (2007) 709-723. doi:10.1109/TPS.2007.896989.

[6] M.L. Walker, D.A. Humphreys, J.A. Leuer, J.R. Ferron, B.G. Penaflor, Implementation of model-based multivariable control on DIII-D, Fusion Eng. Des. 56-57 (2001) 727-731. doi:10.1016/S0920-3796(01)00393-3.

[7] M.L. Walker et al., Advances in integrated plasma control on DIII-D, Fusion Eng. Des. 82 (2007) 1051-1057. doi:10.1016/j.fusengdes.2007.07.047.

[8] T.A. Casper, W.H. Meyer, L.D. Pearlstein, A. Portone, ITER shape controller and transport simulations, Fusion Eng. Des. 83 (2008) 552-556. doi:10.1016/J.FUSENGDES.2007.09.009.

[9] F. Felici et al., Real-time physics-model-based simulation of the current density profile in tokamak plasmas, Nucl. Fusion. 51 (2011) 083052. doi:10.1088/0029$5515 / 51 / 8 / 083052$.

[10] C. Rapson, F. Monaco, M. Reich, J. Stober, W. Treutterer, Simulation of feedback control system for NTM stabilisation in ASDEX Upgrade, Fusion Eng. Des. 88 (2013) 1137-1140.

doi:10.1016/J.FUSENGDES.2013.02.127.

[11] Modelica Association, Modelica and the Modelica Association, (n.d.). https://modelica.org/ (accessed 7 September 2018).

[12] Dassault Systèmes, Dymola, (n.d.). https://www.3ds.com/productsservices/catia/products/dymola (accessed 7 September 2018).

[13] W. Suttrop, L. Höllt, Asdex Upgrade Team, A.U. Team, Predictive Simulation of Tokamak Discharge Behaviour based on Simple Scalings, in: C. Hidalgo, B.P. van Milligen (Eds.), 32nd EPS Conf. Plasma Phys., EPS, Geneva, 2005: p. P-4.076.

http://epsppd.epfl.ch/Tarragona/pdf/P4_076.pdf (accessed 7 September 2018).

[14] J.B. Lister et al., Evolution of the DINA-CH tokamak full discharge simulator, Fusion Eng. Des. 74 (2005) 633637. doi:10.1016/J.FUSENGDES.2005.06.284.

[15] The Mathworks Inc, Simulink - Simulation and ModelBased Design, (n.d.).

https://mathworks.com/products/simulink.html (accessed 7 September 2018).

[16] INRIA, Scicos Homepage, (n.d.). http://www.scicos.org/ (accessed 7 September 2018).

[17] Kepler Collaboration, The Kepler Project, (n.d.). https://kepler-project.org/ (accessed 7 September 2018).
[18] D.A. Humphreys et al., Development of ITER-relevant plasma control solutions at DIII-D, Nucl. Fusion. 47 (2007) 943-951. doi:10.1088/0029-5515/47/8/028.

[19] M.L. Walker et al., A simulation environment for ITER PCS development, Fusion Eng. Des. 89 (2014) 518-522. doi:10.1016/J.FUSENGDES.2014.02.009.

[20] G. Raupp et al., Event generation and simulation of exception handling with the ITER PCSSP, Fusion Eng. Des. 89 (2014) 523-528. doi:10.1016/j.fusengdes.2014.04.068.

[21] M.L. Walker et al., The ITER Plasma Control System Simulation Platform, Fusion Eng. Des. 96 (2015) 716719. doi:10.1016/J.FUSENGDES.2015.01.009.

[22] M.L. Walker, Enabling co-simulation of tokamak plant models and plasma control systems, Fusion Eng. Des. 127 (2018) 60-65. doi:10.1016/J.FUSENGDES.2017.12.021.

[23] F. Janky, E. Fable, W. Treutterer, ASDEX Upgrade Team, AUG flight simulator development, in: Proc. SOFT 2018, to be published at Fusion Engineering and Design, n.d.

[24] W. Treutterer et al., ASDEX Upgrade Discharge Control System-A real-time plasma control framework, Fusion Eng. Des. 89 (2014) 146-154. doi:10.1016/j.fusengdes.2014.01.001.

[25] E. Fable et al., Novel free-boundary equilibrium and transport solver with theory-based models and its validation against ASDEX Upgrade current ramp scenarios, Plasma Phys. Control. Fusion. 55 (2013) 124028. doi:10.1088/0741-3335/55/12/124028.

[26] E. Fable et al., Dynamical coupling between magnetic equilibrium and transport in tokamak scenario modelling, with application to current ramps, Plasma Phys. Control. Fusion. 55 (2013) 074007. doi:10.1088/0741$3335 / 55 / 7 / 074007$.

[27] F. Janky, E. Fable, W. Treutterer, H. Zohm, Simulation of burn control for DEMO using ASTRA coupled with Simulink, Fusion Eng. Des. 123 (2017) 555-558. doi:10.1016/J.FUSENGDES.2017.04.043.

[28] E. Fable et al., Dynamical coupling between magnetic equilibrium and transport in tokamak scenario modelling, with application to current ramps, Plasma Phys. Control. Fusion. 55 (2013) 074007. doi:10.1088/0741$3335 / 55 / 7 / 074007$. 